\title{
Material Behavior under Extreme Domain Constraint in Laser-assisted Surface Nanostructuring
}

\author{
Chong $\mathrm{Li}^{\mathrm{a}}$, Lijun Zhang ${ }^{\mathrm{b}}$, Yan $\mathrm{Li}^{\mathrm{c}}$, and Xinwei Wang ${ }^{\mathrm{a}, \mathrm{d}, ~ *}$ \\ a2010 Black Engineering Building, Department of Mechanical Engineering, \\ Iowa State University, Ames, Iowa 50011, USA \\ bShanghai Ocean University, Shanghai, P.R. China \\ ${ }^{c}$ College of Engineering,Ocean University of China, \\ Qingdao266100,P.R. China \\ ${ }^{\mathrm{d}}$ School of Urban Development and Environmental Engineering, \\ Shanghai Second Polytechnic University, Shanghai 201209, P.R. China
}

\begin{abstract}
Using systematic atomistic modeling, the objective of this study is to explore the nanoscale tip-confinement effect in near-field surface nanostructuring. It is to provide important knowledge base for the understanding and design of tip-based manufacturing at the nanoscale.Our result reveals that the number of ablated-out atoms decreases significantly due to the tip-confinement. Tip apex oscillation occurs because of tip interaction with the substrate. The dynamic nanostructuring process and final surface structure are strongly affected by tip-substrate distance and laser fluence. When the tip-substrate distance is smaller, it takes a longer time for the substrate to cool down and solidify.
\end{abstract}

Keywords: Surface nanostructuring, Tip-confinement, Molecular dynamics, Recrystallization process, Tip distortion

*Corresponding author. Email: xwang3@iastate.edu, Tel: 001-515-294-8023, Fax: 001-515-294-3261

(C) 2015. This manuscript version is made available under the Elsevier user license http://www.elsevier.com/open-access/userlicense/1.0/ 


\section{Introduction}

Surface nanostructuring is an active research area in the last decades because of its promising application in high-resolution nanolithography, ultrahigh density data storage, nanoeletronics, and nanophotonics [1]etc. Nowadays, miniaturization is the trend of the electronic and mechanical devices. However, the traditional maskless manufacturing techniques are challenged by the diffraction limit. To meet the needs in industries, great effort has been devoted to the development of scanning probe microscopy (SPM) techniques[2, 3], which enable ultrahigh surface imaging [4] as well as surface modification at the molecular level[1].

A wide spectrum of microscopy techniques are developed including near-field scanning optical microscopy (NSOM)[5, 6], scanning tunneling microscopy (STM)[7], and atomic force microscopy (AFM)[8, 9]etc. There are two modes of NSOM, the aperture mode and the apertureless mode. The combination of laser illumination and a sharp tip (tungsten tip, probe of AFM etc) yields the apertureless near-field scanning optical microscopy. A resolution as small as $1 \mathrm{~nm}$ has been declared for the apertureless mode[10, 11]. For surface nanostructuring, processing structure down to $10 \mathrm{~nm}$ has been accomplished[12-14].And field enhancement [1519] at thenanoscale and the thermal expansion of tip [20-23] are observed in this apertureless mode. Lu et al. demonstrated that the field enhancement and the mechanical indentation resulting from the AFM tip can be applied effectively in the surface nanostructuring in their works[24, 25].

The combination of laser and AFM tip is not only applied in surface nanostructuring but also in tip-enhanced Raman spectroscopy (TERS). Raman spectrum arises from the vibrational 
frequencies of molecules and providesmaterial information which is particularly valuable in chemistry[26].Combined with the near-field technique, tip enhanced Raman scattering (TERS) [27-31] has become a powerful tool for material analysis at the nanoscale. Considering the close distance between the tip and the substrate which may result in measurement instability, the thermal expansion of the tip and substrate has been studied extensively[32, 33]. Chen et al employed the finite element method (FEM) to simulate the electromagnetic field of the tungsten SPM tip and silicon substrate system under laser irradiation [31]. Tip-substrate distance effect and the optimum tip apex radius for different half taper angles are declared.Yue et al. explored the thermal transport at sub-10 nm scale with different polarization angles of the incident laser in their experiment and simulation[34]. A temperature rise as high as $250^{\circ} \mathrm{C}$ on the silicon substrate for polariation angle of $15^{\circ}$ and $30^{\circ}$ was reported in their work.

Molecular dynamics (MD) simulation ishelpful in understanding the underlying physics from the molecular level. It has been widely applied in the exploration of the physical mechanisms in laser-material interaction[35-38]. Wang [39]studied the thermal and mechanical behavior in surface nanostructuring with laser-assisted STM. The solidification and epitaxial growth [40], the molecular weight and density of the ambient gas [41] have also been studied in surface nanostructuring. Feng et al.[42] explored the shock wave in near-field laser material interaction and the kinetic and physical properties of the shock wave was revealed.

The aforementioned MD simulation works provide great understanding of the physical phenomena of melting, crystallization and the environment effect. However, they all exclude the effect of the tip-confinement. Considering the close distance between the SPM tip and the 
substrate which is at the molecular level, the effect of the spatial confinement of the tip apex may result in more complicated physical phenomena in surface nanostructuring. Also this knowledge will help understand how and to what extent the tip apex could be damaged by surface nanostructuring. In this work, a nanoscale size tip needle is included to study the tip-confinement effect in the surface nanostructuring process. The tip-substrate distance and the laser fluence's effect are studied in detail. Also tip-apex distortion is studied under different nanostructuring conditions.

\section{Methodologies of Simulation}

Argon is chosen as the material in this simulation work, due to its great computational efficiency. This is critical for this work since our modeled system is composed of a large number of atoms and the whole physical process under simulation is very long (close to $5 \mathrm{~ns}$ ). Argon crystal is arranged in the simple face-centered cubic structure and well described by the LennardJones (12-6) potential.It is easy for the modeling and fast for the computation while the conclusion does not lose its generality. A lot of works in our group [35-38] [43, 44] have proved its validity in laser-material interaction simulation.The LJ potential well depth $\varepsilon$ is $0.0103 \mathrm{ev}$ and the equilibrium separation parameter $\sigma$ is $3.406 \AA$. And the cut-off distance is set to $2.5 \sigma$. The energy is calculated as Lennard-Jones potential within the cut-off distance and is viewed as zero outside the cut-off distance. LAMMPS is employed in this work[45].

Figure 1(a) shows the physical model of the simulation and the domain construction. To save the computation cost, a quasi-3D model is adopted. The thickness of the whole simulation system (including the tip and the substrate) in the $y$ direction is $10 \mathrm{~nm}$ : relatively small in scale 
compared with $x$ and $z$ direction size. Periodic boundary conditions are applied in the $x$ and $y$ directions. The boundary condition in the $z$ direction is the fixed boundary condition. The substratesize is100 $\mathrm{nm} \times 10 \mathrm{~nm} \times 30 \mathrm{~nm}(x \times y \times z)$. The interaction of substrate-substrate atoms obeys the Lennard-Jones (12-6) potential.

There are three important parameters for the tip construction: apex radius $R_{t i p}=15$ nm,half taper angle $\theta=10^{\circ}$, and tip length $L=114 \mathrm{~nm}$. The tip thickness is also $10 \mathrm{~nm}$ in the $y$ direction. The atoms inside the tip at $z \geq 98.1 \mathrm{~nm}$ are removed and the actual shape in the regionz $\geq 98.1 \mathrm{~nm}$ is an empty cell surrounded by the fivewalls $(x, y$ direction and $z$ direction on the top) with a thickness of $1 \mathrm{~nm}$. The tip atoms in the region $z \geq 97.1 \mathrm{~nm}$ are set static. They do not move in the simulation. Since we are interested in the tip area close to the substrate, this simplification is not going to affect the final results and analysis. The tip is created based on the argon crystal. The interaction of tip-tip atoms are described by the LJ (12-6) potential as mentioned before. However, as to the interaction of the tip atoms and the substrate atoms,only the repulsive component is considered and the attractive force is neglected. And since the shock wave phenomenon $[36,44,46]$ has been observed even at the nanoscale and the behavior of the tip and shock wave may be strongly changed due to their interaction, it is necessary to introduce ambient gas atoms [red dots in Fig. 1(a)] in this model. Only the repulsive force is considered for the gas-gas, gas-tip and gas-substrate atoms interaction. More details can be foundin our previous works.[36, 44] The gas pressure is $0.22 \mathrm{MPa}$ in the thermal equilibrium state at $T=50 \mathrm{~K}$.

In the experiment, the substrate is placed underneath the tip. The incident laser irradiates the AFM tip in a certain polarization angle (the angle to the tip axis) which can be adjusted in the 
experiment. The tip serves as a receiving antenna to collect the laser energy as well as an emitting antenna which results in the enhancement of an optical field at the nanoscale[34]. For the MD simulation, the antenna effect of the tip is not easy to be realized. Since the laser absorption in the tip is ignored in this work and the emphasis is placed on the near-field heating of the substrate, the laser irradiation is focused on a circular spot with a radius of $2 \mathrm{~nm}$ on the substrate. Figure 1(b) shows the laser beam intensity distribution. A single laser pulse of $40 \mathrm{ps}$ is applied in this simulation work. The laser energy is spatially uniform on the spot. The full width at half maximum (FWHM) of the incident laser beam intensity distribution is 11.5 ps and the peak occurs at $t=9 \mathrm{ps}$. Laser energy absorption obeys the Beer-Lambert law. To accomplish it, the laser absorption region is divided into a number of bins of thickness $\Delta z=1 \mathrm{~nm}$. And the artificial absorption depth of $5 \mathrm{~nm}$ is used in our work. The incident laser energy decreased exponentially after the absorption in each bin. The details have been discussed in our previous works[36, 44, 47].

After constructing the whole physical domain for modeling and definition of atom interaction and boundary conditions, the system is at first treated as a canonical ensemble (NVT) and modeled for 1 ns at $T=50$ Kand then a microcanonical ensemble (NVE) for 800 ps to reach thermal equilibrium. Then the laser energy is applied on the substrate surface. After that, the whole system is in NVE again for recrystallization. During the laser irradiation and in the early stage of laser ablation for all cases except for the case $E=5 \mathrm{~J} / \mathrm{m}^{2}, d=2 \mathrm{~nm}$, the time step is set to $2 \mathrm{fs}$. Other than that, the time step is set to 5 fs. For the case $E=5 \mathrm{~J} / \mathrm{m}^{2}, d=2 \mathrm{~nm}$, the time step is 2 fs all the time. 


\section{Results and Discussion}

\subsection{Material behavior under extreme domain confinement: a general picture}

Surface nanostructuring is affected by the incident laser(including the laser fluence $E$ and the polarization angle), the tip-substrate distance $d$, and the substrate material properties. In this modeling, different laser fluence $E$ and various tip-substrate distance $d$ are used to study their effects. The influence of these two factorsis going to be discussed in the next several sections. In this section, the case of laser fluence $E=5 \mathrm{~J} / \mathrm{m}^{2}$, and tip-substrate distance $d=2 \mathrm{~nm}$ is chosen to demonstrate the general pictures of laser ablation and recrystallization process when tipconfinement is considered.

Figure (2) is the atomic snapshot at different times. Laser energy is applied from $t=0 \mathrm{ps}$. It is clearly shown that the ablation has already started at $t=10 \mathrm{ps}$. The tip apex is also affected by the ablation from the substrate. Without the tip-confinement, the ablated substrate material expands freely in the space and it has been studied in detail in our previous works.[36, 44] In this nanostructuring, the ablated materials attack the tip apex in high speed. Due to the close distance and the high speed of the ejected plume, the structure of the tip apex is distorted and melts as shown at $t=20 \mathrm{ps}$. The dark region in the apex indicates the hot status of the small part in the tip. And the destruction is not permanent since the region under destruction disappeared and no voids are formed afterwards.

Even though the laser pulse is very short, the thermal expansion lasts a relatively long time after the laser pulse. Figures from $t=10 \mathrm{ps}$ to $t=100 \mathrm{ps}$ show the thermal expansion and relaxation of the substrate. From $t=200 \mathrm{ps}$ to the end of the simulation $t=2580 \mathrm{ps}$, 
recrystallization is observed. Because of the strong stress wave in the substrate, the structure of the material is distorted from the well-arranged crystal structure as shown at $t=100 \mathrm{ps}$. From the atomic configuration, it is seen that most of the damages are temporary and it is back to the good crystal structure again in the recrystallization process. This can be confirmed by the crystallinity study discussed later. However, the destruction in the laser irradiation region is permanent. At the end of simulation at $t=2580 \mathrm{ps}$, a cone-shape crater forms. Meanwhile, distortion close to the bottom in the substrateis also observed.

Figure 3 is for the temperature contours corresponding to the nanostructuring process in Fig. 2. Initially, the thermal equilibrium at $T=50 \mathrm{~K}$ of the whole system is achieved before the laser irradiation. Laser irradiation starts at $t=0$ ps. A hot spot which is above $320 \mathrm{~K}$ is observed at $t=10 \mathrm{ps}$ underneath the tip. From $t=10 \mathrm{ps}$ to $t=40 \mathrm{ps}$, the region of high temperature expands because of the heat transfer from the irradiation spot to the surroundings. And at the same time, laser ablation is observed. However, the ablation is prohibited from expanding in space by the tip. Shock waves with a high temperature front are shown in the Fig. 3 at $t=100 \mathrm{ps}$. The temperature of the substrate drops continuously from 100 ps to 2580 ps. By the end of the simulation $t=2580$ ps, the temperature of the substrateis close to $50 \mathrm{~K}$ again.

Figure 4 shows the atom number density contours for the case in Fig. 2. To get the contour, the whole domain is divided into $1 \times 10.2 \times 1(x \times y \times z) \mathrm{nm}^{3}$ cells. In the part irradiated by the high energy laser, the atom number density decreases during the laser irradiation as shown from $t=10$ ps- 40 ps. Argon lattice constant differs from the cell size in the contours. Consequently, the atom number density in the cells changes periodically. The contours show 
patterned blocks of red and dark color and this periodicity is very helpful in viewing the structure distortion. As the thermal energy dissipates towards the bottom and the surroundings, recrystallization occurs. From $t=200$ ps, the atom number density increases continuously in the region of laser irradiation. Noticeably, there is distortion in the atom number density contour in the region of defects at $t=2580 \mathrm{ps}$. The final defects are the structure distortion effect.

The laser irradiation leads to the structure destruction of the substrate and distortion of the tip apex. Crystallinity is a good parameter to describe the crystal structure destruction and it is defined as $[37,40]$

$$
j\left(r_{j, x}\right)=\frac{1}{N}\left|\sum_{j} e^{i 2 \pi\left(2 r_{j, x} / a\right)}\right|,
$$

where $r_{j, x}$ is the $x$ coordinate of atom $j$ and $a$ is the lattice constant.The summation is done over all atoms in each cell whose size is $1 \times 10.2 \times 1(x \times y \times z) \mathrm{nm}^{3}$ in this work.The crystallinity value ranges from 0 to 1 . If atoms are regularly distributed in space with their spacing in the $x$ direction equal to $n(a / 2)$, the function will be equal to 1 . In the amorphous state, the function $\phi\left(r_{j, x}\right)$ will be much less than 1 , in fact very small (close to zero).

Figure 5 is the crystallinity contours for the case $E=5 \mathrm{~J} / \mathrm{m}^{2}, d=2 \mathrm{~nm}$. Before laser irradiation, both the tip and the substrate are in good crystal structure and their crystallinity value is very close to 1 . Because of the laser irradiation, the melting region expands from $t=10 \mathrm{ps}$ to $t=$ $40 \mathrm{ps}$. The crystallinity value in the melting region is low and very close to 0 . The crystallinity of the tip apex also drops because of its interaction with the exploded substrate plume. The distorted solid materialregion expands towards the substrate bottom as well as the in the radial direction afterwards. So the distorted solid materialregion becomes wider and deeper from $t=10 \mathrm{ps}$ to $t=$ 
100 ps. From $t=200$ ps to $t=2060$ ps, the distortedsolid material region shrinks. Protrusions show up at $t=1000 \mathrm{ps}$ in the recrystallization process. At the end of the simulation, the crystallinity of both the tip and the substrate is close to 1 again which indicates good crystal structure at the steady state.Distortion in the substrates at the final state can be readily identified since they have a low crystallinity value (0.5 0.7), as shown in the figure at $t=2580 \mathrm{ps}$.

\subsection{Effect of constraint domain size}

When the laser fluence is above the material ablation threshold, material phase explosion occurs. Systematic studies have been done to understand the plume and thermal and stress issues in laser ablation in vacuum[49, 50].The ablated material explodes and expands into the open space with a high velocity. When an ambient gas is included in the analysis model, a shock wave is formed. Its formation and propagation has been explored in our previous works[36, 44].

Here the tip-confinement effect is considered and studied in detail. In Fig. 6, the comparison of the case with free space and the ones with tip is made. The laser irradiates the substrate at first. Afterwards, laser ablation happens and shock wave forms. For the tip-free case, the ablated material can move out freely in the ambient gas and a strong shock wave is formed. Meanwhile, there are several clusters in the region $40 \mathrm{~nm}<\mathrm{z}<100 \mathrm{~nm}$ in the tip-free case. However, there are no clusters in the cases with tip-confinement because of the repulsive force from the tip in the near-field. And the expansion of the laser ablation is blocked by the tip. The shock wave is attenuated as well when the tip confinement is included. With the increase of the tip-substrate distance $d$, the constraint of the tip on the substrate material explosion becomes less

and less. As a result, the shock wave resulting from the laser ablation becomes stronger and 
stronger.

Correspondingly, the comparison of the snapshots at $t=100$ ps for $d=2,5$ and $7 \mathrm{~nm}$ demonstrates that the tip apex distortion is less severe with the increase of the tip-substrate distance. Moreover, the recrystallization process also shows some differences. At $t=1000 \mathrm{ps,} \mathrm{the}$ profile of the melting region is different. Due to the interaction of the tip and the substrate, the melting region with $d=2 \mathrm{~nm}$ is more widely open than the cases of $d=5 \mathrm{~nm}$ and $7 \mathrm{~nm}$. For the tip-free case, the melting region is very narrow.It is conclusive that the constraint by the tip also slows down the recrystallization process in substrate after laser heating since the confinement prevents high energy atoms from flying out.

Figure 7(a) is the cone-shape crater profile at the end of the simulation for different tipsubstrate distance scenarios. The depth is not affected by the tip-substrate distance $d$ too much. However, the protrusion around the crater shows different profiles. For the free space case, the protrusion height is lower and the width of the protrusion is smaller. For the cases of $d=2 \mathrm{~nm}$ and $d=5 \mathrm{~nm}$, the protrusion and the crater match with each other very well. However, for the case $d=7 \mathrm{~nm}$, the protrusion height is a little bit lower and the width becomes wider. The crater emerges first because part of the substrate material is removed in the laser ablation region. After laser ablation, part of the ejected substrate atoms move back towards the substrate because of the interaction with the ambient gas and the tip.

To understand how the exploded substrate plume is affected by the tip constraint, the number of the atoms that are above $z=34.5 \mathrm{~nm}$ at different instantsare studied [shown in Fig. 
7(b)]. In the beginning, the laser ablation is featured with a number of atoms ejected from the substrate. The effect of the tip-constraint is outstanding. The tip-free case has more atoms ejected into the space $z>34.5 \mathrm{~nm}$. Due to the tip-confinement, the atom number above $z=34.5 \mathrm{~nm}$ is down to 4000 for $d=1 \mathrm{~nm}$ from 10000 for tip-free case. For the tip-free case, the number of flying-out atoms in the final state is 3982 , accounting for $0.5 \%$ of the total number of the substrate atoms. Due to the constraint effect of the tip, the atom number in the case $d=7 \mathrm{~nm}$ is 575 which is only $15 \%$ of the tip-free case. Furthermore, the number of flying-out atoms decreases with the decrease of the tip-substrate distance. As $d=2 \mathrm{~nm}$, the flying-out atom number in the final steady state is only 198 , less than $5 \%$ of the tip-free case.

Because the laser fluence is the same for all cases shown in Fig. 7(a), the crater shape in Fig.7(a) does not differ too much for all cases. The crater depth is controlled by the laser fluence while the side profile of the crater is slightly affected by the tip confinement. The change of tipsubstrate distance does not make much difference to the side profile of the crater. However, the tip-substrate distance affects the shape of the protrusion. First, the tip-confinement affects the number of the atoms flying into the free space as discussed above. Second, the tip-confinement changes the trajectories of the ejected clusters. As shown in Fig.7(b), there is oscillation due to the interaction between the tip and the substrate. The oscillation is very strong for $d=1 \mathrm{~nm}$ and $d=2 \mathrm{~nm}$ since the tip-substrate distance is very small. Most of the clusters deposit on the surface close to the crater for the small tip-substrate distance. As for the tip-free case, the ejected atoms are spread in a wide region. So the protrusion is small and consists of a small number of atoms. For the case with a small tip-substrate distance, the ejected atoms deposit on the surface close to the crater. Consequently, the protrusion is higher. By increasing the distance $d$, the deposition 
region move a little bit further away. That is the reason why the protrusion width is bigger for the case of $d=7 \mathrm{~nm}$.

Figures $8(a)$ and (b) show the bottom point of the crater $\left(Z_{b}\right)$ and the solid-liquidinterface bottompoint $\left(Z_{s l}\right)$ in the substrate. In the early stage of melting, both $Z_{b}$ and $Z_{s l}$ go down very quickly [as shown in the insets of Figs. 8(a) and (b)]. The crater bottommoves up quickly after the laser ablation, while the solid-liquid interface goes up slowersince this process is controlled by heat conduction and solidification. For the tip-free and $d=7 \mathrm{~nm}$ cases, the crater bottom point is the lowest at $t=500$ ps when it is still in the recrystallization process. For the $d=1 \mathrm{~nm}$ case, the crater bottom point at $t=500 \mathrm{ps}$ is the highest. The interaction between the tip and the substrate leads to the small number of ejected atoms and the change of the melting interface shape. The interface is round for the case with $d=1 \mathrm{~nm}$, while the crater bottom is relatively sharp for the tip-free case. As a result, the crater bottom point differs for different cases. The interaction between the tip and the substrate also results in the relatively slow recrystallization process considering the atoms with high energy are prohibited from moving out. This is also observed in Fig. 6. The solid-liquid interfacemoves up in the fastest speed for the tip-free case. Then it is the $d=7 \mathrm{~nm}$. Around $t=2000 \mathrm{ps}$, the recrystallization process is finished for laser fluence $E=5 \mathrm{~J} / \mathrm{m}^{2}$ of various tip-substrate distance. The solid-liquid interface point is gone and a stable coneshaped crater forms. The crater bottom points of different cases in the final state are very close to each other.

Given the fact that only one single laser pulse is considered in this work for simplicity, it is expected that the final surface structure could be quite different if multiple laser pulses are 
used in the experiment, especially for small tip-substrate distance configurations.

\subsection{Effect of laser energy on surface nanostructuring}

Both the tip-substrate distance and the laser fluence play important roles in nanostructuring dynamic process.Besides various tip-substrate distance, different laser fluences $\left(E=2 \mathrm{~J} / \mathrm{m}^{2}, 5 \mathrm{~J} / \mathrm{m}^{2}, 10 \mathrm{~J} / \mathrm{m}^{2}\right)$ are also studied in this work. The laser power affects the melting depth and the laser ablation process. Figure 9 is the snapshots and crystallinity contours under different laser fluence in the final simulation state. The crater size of the laser fluence $E=2$ $\mathrm{J} / \mathrm{m}^{2}$ is much smaller than that of the laser fluence $E=5 \mathrm{~J} / \mathrm{m}^{2}$ and $10 \mathrm{~J} / \mathrm{m}^{2}$.The distortion position of the low laser fluence case $\left(E=2 \mathrm{~J} / \mathrm{m}^{2}\right)$ is much higher than that of the high laser fluence $(E=5$ $\mathrm{J} / \mathrm{m}^{2}, 10 \mathrm{~J} / \mathrm{m}^{2}$ ). Large-size distortion occurs in the case with $E=10 \mathrm{~J} / \mathrm{m}^{2}$. Owing to the high laser energy, even after a long time recrystallization, the part near the crater is still in low crystallinity value which features bad crystal structure.Two factors may account for the low crystallinity. First, nanograins with different orientations may form in the resolidification process. Second, the system size is not big enough and the simulation time may not be long enough. If the simulation system is large and the simulation time is long enough, the crystallinity value in the high laser fluence case is expected to get close to the low laser fluence case. But full recovery is not expected for the distortion. In work [40], a large system consisting of more than $1 \times 10^{8}$ atoms was studied for laser ablation and cooling. After a long simulation time ( 2 ns), the distortion could still be seen clearly.

Figure 10(a) shows the crater profile for two different fluences. The width and depth of crater for the case $E=2 \mathrm{~J} / \mathrm{m}^{2}$ is smaller than that of the case $E=5 \mathrm{~J} / \mathrm{m}^{2}$. The protrusion size is also 
smaller for the low laser energy case. When the laser fluence is decreased, the atom number ejected from the substrate is also decreasing in the ablation. Consequently, a crater of smaller size is formed and the protrusion around the crater is also smaller.Figure 10(b) demonstrates that the solid-liquid interface bottom point $Z_{s l}$ is higher for $E=2 \mathrm{~J} / \mathrm{m}^{2}$. This accounts for the location change of the distortion as shown in Fig. 9(a). For the small laser fluence, the melting depth is small and $Z_{s}$ is closer to the upper substrate surface. And the effect of the laser fluence on the bottom part of the substrate is small. As a result, the distortion part is close to the upper surface. Otherwise it is close to the bottom part. The high laser fluence also effectively increases the time of crystallization. It takes 1800 ps for the case $E=5 \mathrm{~J} / \mathrm{m}^{2}$ to get fully recrystallized. It is almost the 2 times of that for the case $E=2 \mathrm{~J} / \mathrm{m}^{2}$.

\subsection{Tip distortion by the domain constraint}

The tip is affected by the laser fluence as well. In SPM-based nanomanufacturing, this effect is a big concern, and usually requires frequent replacement of the SPM tip after a certain time of use. Figure 11(a) shows the effect of different laser fluence on the tip. They are the snapshots at $t=1440 \mathrm{ps}$. The tip apex is distorted seriously under the laser fluence of $E=10 \mathrm{~J} / \mathrm{m}^{2}$. The shape of the tip apex for the case $E=10 \mathrm{~J} / \mathrm{m}^{2}$ is more blunt than the case $E=5 \mathrm{~J} / \mathrm{m}^{2}$. Tip wear and thermal expansion is always a concern of researchers in the nanostructuring process. In Fig.9(a), the tip apex is reshaped and blunt even after recrystallization for almost 5 ns when $E=$ $10 \mathrm{~J} / \mathrm{m}^{2}$. However, the simulation results show that it is not only related to the laser fluence. The tip-substrate distance is also important for the tip distortion. Figure 11(b) shows the tip distortion at $t=40 \mathrm{ps}$ for different tip-substrate distance. For the case with tip-substrate distance $d=2 \mathrm{~nm}$, the degree of distortion is more than that of the tip-substrate distance $d=7 \mathrm{~nm}$. It is readily to 
understand that the interaction between the tip and the substrate is stronger when the distance is smaller. This stronger interaction leadsto more severe tip apex damage and distortion.

Besides permanent apex distortion, we also observe oscillation of the tip apex during surface nanostructuring due to the interaction with the substrate. Figure 12 shows the movement of the tip apex. The oscillation of the tip is clearly observed. Both the substrate and tip come to strong contact right after the material explosion, impact each other strongly, then separate. This induces oscillation/vibration in both. Also we observe the "bending" effect of the tip because of the strong impact from the ablated substrate.And the oscillation amplitude is big for the close tipsubstrate distance since the interaction between the tip and the substrate is attenuated if the tip and substrate distance increases. The ablation weakens if the laser fluence is decreased. That is why the oscillation for the case $E=2 \mathrm{~J} / \mathrm{m}^{2}$ is negligible while the oscillation in the case of $E=5$ $\mathrm{J} / \mathrm{m}^{2}$ is strong.

To have a deep insight of the constraint effect of the tip on the exploded plume dynamics, a window of size of $4 \times 10 \times 2 \mathrm{~nm}^{3}(x \times y \times z)$ beneath the tip is chosen for temperature monitoring to study the constraint effect. For all the cases, the temperature in this window increases at first because of the laser ablation. Then the temperature decreases after around $t=30 \mathrm{ps}$. This is because at the later time, the ablated material deposited on the surface or they fly away from this window. When the tip-sample distance $d$ is as small as $1 \mathrm{~nm}$, the window temperature is higher than the free case during the laser ablation ( $t<50 \mathrm{ps)}$. The tip sets up the spatial confinement for the spread of the ablated material. When the tip-sample distance is small, the ablated material is trapped in the near field for a long time. That explains why the temperature in the window for $d=$ 
1nm can reach a higher value.

\section{Conclusions}

In this work, systematic atomistic modeling has been conducted to understand the tipconfinement effect on surface nanostructuring. Material ablation was trapped by the tip and a significant decrease of the number of atoms flying out of the surface was observed. For tip-free laser-induced surface ablation, large atom clusters were observed in our reference modeling. However, for tip-confined laser-ablation, no such clusters were observed. Tip oscillation occurred because of the tip interaction with the substrate during laser ablation. The profile of the cone-shape crater was not affected by the tip substrate distance too much. The laser fluence played the dominant role in the crater characterization. The protrusion around the crater was affected by the tip-substrate distance and the laser fluence.The recrystallization process was significantly influenced by the tip-confinement. The recrystallization time was longer for the case with stronger tip confinement. This is because of the interaction between the tip and the substrate: the confinement prevents high energy atoms from flying out. As a result, more laser energy was left to the substrate. The tip apex was distorted during the laser ablation. Both the tipsubstrate distance and the laser fluence played import roles in the distortion. For the case of $E=$ $10 \mathrm{~J} / \mathrm{m}^{2}$, the tip apex was reshaped to be blunt. In tip-based laser surface nanostructuring, careful control of the tip-substrate distance and laser energy becomes critical to maintain a sound tipapex geometry for both structure quality control and prolonged life time of the tip before it gives intolerable surface nanostructures. 


\section{Acknowledgements}

Support of this work by the National Science Foundation (CMMI-1029072 and CMMI1200397) is gratefully acknowledged. X.W. also thanks the partial support of the "Eastern Scholar" program of Shanghai, China. 


\section{References}

1. A.Chimmalgi , C.P. Grigoropoulos, and K. Komvopoulos, Surface nanostructuring by nano/femtosecond laser-assisted scanning force microscopy. Journal of Applied Physics, 2005. 97(10): p. 104319.

2. R. Huber, M. Koch, and J. Feldmann, Laser-induced thermal expansion of a scanning tunneling microscope tip measured with an atomic force microscope cantilever. Applied Physics Letters, 1998. 73(17): p. 2521-2523.

3. Yasushi Inouye and Satoshi Kawata, Near-field scanning optical microscope with a metallic probetip. Optics Letters, 1994. 19(3): p. 159-161.

4. Neil Anderson, Pascal Anger, Achim Hartschuh, and Lukas Novotny,Subsurface Raman Imaging with Nanoscale Resolution. Nano Letters, 2006. 6(4): p. 744-749.

5. Vladimir V. Protasenko and Alan C. Gallagher, Apertureless Near-Field Scanning Optical Microscopy of Single Molecules. Nano Letters, 2004. 4(7): p. 1329-1332.

6. Gerd Kaupp, Andreas Herrmann, and Michael Haak, Artifacts in scanning near-field optical microscopy (SNOM) due to deficient tips. Journal of Physical Organic Chemistry, 1999. 12(11): p. 797-807.

7. Stefan Grafström, Photoassisted scanning tunneling microscopy. Journal of Applied Physics, 2002. 91(4): p. 1717-1753.

8. G. Binnig, C.F. Quate, and Ch. Gerber, Atomic Force Microscope. Physical Review Letters, 1986. 56(9): p. 930-933.

9. $\quad$ E. Meyer, Atomic force microscopy. Progress in Surface Science, 1992. 41(1): p. 3-49.

10. J. Koglin, U.C. Fischer, and H. Fuchs, Material contrast in scanning near-field optical microscopy at 1-10 nm resolution.Physical Review B, 1997. 55(12): p. 7977-7984.

11. F. Zenhausern, Y. Martin, and H.K. Wickramasinghe, Scanning Interferometric Apertureless Microscopy: Optical Imaging at 10 Angstrom Resolution. Science, 1995. 269(5227): p. 1083- 
1085.

12. J. Jersch, F. Demming, and K. Dickmann, Nanostructuring with laser radiation in the nearfield of a tip from a scanning force microscope. Applied Physics A, 1997. 64(1): p. 29-32.

13. Huang, S.M., M. H. Hong, Y. F. Lu, B. S. Lukỳanchuk, W. D. Song, and T. C. Chong, Pulsedlaser assisted nanopatterning of metallic layers combined with atomic force microscopy. Journal of Applied Physics, 2002. 91(5): p. 3268-3274.

14. G N Makarov, Laser applications in nanotechnology: nanofabrication using laser ablation and laser nanolithography. Physics-Uspekhi, 2013.56(7): p. 643-682.

15. RenaudBachelot, et al., Apertureless near-field optical microscopy: A study of the local tip field enhancement using photosensitive azobenzene-containing films. Journal of Applied Physics, 2003. 94(3): p. 2060-2072.

16. Yves C. Martin, Hendrik F. Hamann, and H.Kumar Wickramasinghe, Strength of the electric field in apertureless near-field optical microscopy. Journal of Applied Physics, 2001. 89(10): p. 5774-5778.

17. John L. Bohn, D.J. Nesbitt, and A. Gallagher, Field enhancement in apertureless near-field scanning optical microscopy. Journal of the Optical Society of America A, 2001. 18(12): p. 29983006.

18. Xiaoduan Tang, Yannan Yue, Xiangwen Chen, and Xinwei Wang, Sub-wavelength temperature probing in near-field laser heating by particles. Optics Express, 2012. 20(13): p. 14152-14167.

19. Yong-Fengeng Lu, Zhi-HongMai andWai-Kin Chim, Electromagnetic Calculations of the Near Field of a Tip under Polarized Laser Irradiation. Japan. J. Appl. Phys., 1999. 38: p. 5910-5915.

20. J. Boneberg, H.-J. Münzer, M. Tresp, M. Ochmann, P. Leiderer, The mechanism of nanostructuring upon nanosecond laser irradiation of a STM tip. Applied Physics A, 1998. 67(4): p. 381-384.

21. V. A. Ukraintsev and J.T. Yates, Nanosecond laser induced single atom deposition with nanometer spatial resolution using a STM. Journal of Applied Physics, 1996. 80(5): p. 2561-2571. 
22. I. Lyubinetsky, Z. Dohnálek, V. A. Ukraintsev, and J. T. Yates Jr.,Transient tunneling current in laser-assisted scanning tunneling microscopy. Journal of Applied Physics, 1997. 82(8): p. 41154117.

23. Ch. Lienau, A. Richter, and T. Elsaesser, Light-induced expansion of fiber tips in near-field scanning optical microscopy. Applied Physics Letters, 1996. 69(3): p. 325-327.

24. Yong-Feng, L., et al., Laser-Scanning Probe Microscope Based Nanoprocessing of Electronics Materials. Japanese Journal of Applied Physics, 2001. 40(6S): p. 4395.

25. Y. F. Lu, Z. H. Mai, Y. W. Zheng, and W. D. Song, Nanostructure fabrication using pulsed lasers in combination with a scanning tunneling microscope: Mechanism investigation. Applied Physics Letters, 2000. 76(9): p. 1200.-1202

26. Z. Q Tian,, B. Ren, and B.W. Mao, Extending Surface Raman Spectroscopy to Transition Metal Surfaces for Practical Applications. 1. Vibrational Properties of Thiocyanate and Carbon Monoxide Adsorbed on Electrochemically Activated Platinum Surfaces. The Journal of Physical Chemistry B, 1997. 101(8): p. 1338-1346.

27. Mark S. Anderson, Locally enhanced Raman spectroscopy with an atomic force microscope. Applied Physics Letters, 2000. 76(21): p. 3130-3132.

28. Elena Bailoand Volker Deckert, Tip-enhanced Raman scattering. Chemical Society Reviews, 2008. 37(5): p. 921-930.

29. Andrew Downes, Donald Salter, and Alistair Elfick, Finite Element Simulations of Tip-Enhanced Raman and Fluorescence Spectroscopy. The Journal of Physical Chemistry B, 2006. 110(13): p. 6692-6698.

30. Pavel I. Geshev, Ulrich Fischer, and Harald Fuchs, Calculation of tip enhanced Raman scattering caused by nanoparticle plasmons acting on a molecule placed near a metallic film. Physical Review B, 2010. 81(12).

31. Xiangwen Chen and Xinwei Wang, Near-field thermal transport in a nanotip under laser irradiation. Nanotechnology, 2011. 22(7): p. 075204. 
32. Alexander A. Milner,Kaiyin Zhang, Valery Garmider, Yehiam Prior', Heating of an Atomic Force Microscope tip by femtosecond laser pulses. Applied Physics A, 2010. 99(1): p. 1-8.

33. Brendan McCarthy, Yanming Zhao, Ranjan Grover, and Dror Sarid, Enhanced Raman scattering for temperature measurement of a laser-heated atomic force microscope tip. Applied Physics Letters, 2005. 86(11): p. 111914.

34. Yanan Yue, Y., Xiangwen Chen, and Xinwei Wang, Noncontact Sub-10 nm Temperature Measurement in Near-Field Laser Heating. ACS Nano, 2011. 5(6): p. 4466-4475.

35. Xinwei Wang, Xianfan Xu, Molecular Dynamics Simulation of Heat Transfer and Phase Change During Laser Material Interaction. Journal of Heat Transfer, 2002. 124(2): p. 265-274.

36. Chong Li, Jingchao Zhang, and Xinwei Wang, Phase change and stress wave in picosecond laser-material interaction with shock wave formation. Applied Physics A, 2013. 112(3): p. 677687.

37. Chong Li, Kelsey Burney, Kevin Bergler, Xinwei Wang, Structural evolution of nanoparticles under picosecond stress wave consolidation. Computational Materials Science, 2014. 95: p. 7483.

38. Chong $\mathrm{Li}$, Jianmei Wang, and Xinwei Wang, Shock wave confinement-induced plume temperature increase in laser-induced breakdown spectroscopy. Physics Letters A, 2014. 378(45): p. 3319-3325.

39. Xinwei Wang, Large-scale molecular dynamics simulation of surface nanostructuring with a laser-assisted scanning tunnelling microscope. Journal of Physics D: Applied Physics, 2005. 38(11): p. 1805-1823.

40. Xinwei Wang, Yongfeng Lu, Solidification and epitaxial regrowth in surface nanostructuring with laser-assisted scanning tunneling microscope. Journal of Applied Physics, 2005. 98(11): p. 114304.

41. Liying Guo and Xinwei Wang,Effect of molecular weight and density of ambient gas on shock wave in laser-induced surface nanostructuring. Journal of Physics D: Applied Physics, 2009. 
42(1): p. 015307.

42. Xuhui Feng, Xinwei Wang,Nanodomain shock wave in near-field laser-material interaction. Physics Letters A, 2007. 369(4): p. 323-327.

43. Sobieslaw Gacek,Xinwei Wang,Plume splitting in pico-second laser-material interaction under the influence of shock wave. Physics Letters A, 2009. 373(37): p. 3342-3349.

44. Sobieslaw Gacek, Xinwei Wang, Dynamics evolution of shock waves in laser-material interaction. Applied Physics A, 2009. 94(3): p. 675-690.

45. Steve Plimpton,Fast Parallel Algorithms for Short-Range Molecular Dynamics. Journal of Computational Physics, 1995. 117(1): p. 1-19.

46. Cristian Porneala and David A. Willis, Observation of nanosecond laser-induced phase explosion in aluminum. Applied Physics Letters, 2006. 89(21): p. 211121.

47. Xuhui Feng, Xinwei Wang, Effects of laser fluence on near-field surface nanostructuring. Applied Surface Science, 2008. 254(13): p. 4201-4210.

48. Leonid V.Zhigilei, and Barbara J. Garrison, Pressure Waves in Microscopic Simulations of Laser Ablation Leonid. MRS Online Proceedings Library, 1999. 538: p. 491-496.

49. Leonid V.Zhigilei, and Barbara J. Garrison, Microscopic mechanisms of laser ablation of organic solids in the thermal and stress confinement irradiation regimes. Journal of Applied Physics, 2000. 88(3): p. 1281-1298.

50. L.V. Zhigilei, Dynamics of the plume formation and parameters of the ejected clusters in shortpulse laser ablation. Applied Physics A: Materials Science \& Processing, 2003. 76(3): p. 339-350. 


\section{List of Figures}

Figure 1 (a) Physical model for simulating the tip-substrate nanostructuring process. The laser energy is focusedto a circular region with $R_{\text {sub }}=2 \mathrm{~nm}$ in the substrate underneath the tip apex. The substrate is of size $100 \mathrm{~nm} \times 10 \mathrm{~nm} \times 30 \mathrm{~nm}(x \times y \times z)$. There are 3 important parameters for the tip construction: apex radius $R_{t i p}=15 \mathrm{~nm}$,half taper angle $\theta=10^{\circ}$, length of tip $L=114 \mathrm{~nm}$. The atoms inside the tip at $\mathrm{z} \geq 98.1 \mathrm{~nm}$ are removed and the actual shape in the regionz $\geq 98.1 \mathrm{~nm}$ is an empty cell surrounded by 5 walls ( $x, y$ direction and $z$ direction on the top) with a thickness of $1 \mathrm{~nm}$. (b) Laser beam intensity distribution. The full width at half maximum (FWHM) of the incident laser beam intensity distribution is 11.5 ps and peak occurs at $t=9$ ps. (c) Profile of the laser spot. The spot is round with a radius of $R_{\text {sub }}=2 \mathrm{~nm}$.

Figure 2 Snapshots of the case $E=5 \mathrm{~J} / \mathrm{m}^{2}, d=2 \mathrm{~nm}$. Laser ablation starts from around $t=10 \mathrm{ps}$. Because of the confinement by the tip, the ablated material is prevented from moving freely in space. The apex of the tip is slightly distorted by the ablated-out substrate. Recrystallization ( $t=200-2060$ ps) is observed following the ablation. Finally, a steady cone-shape crater is formed. And at the same time, there are defects in the final sample structure at the bottom, as marked in the figure at $t=2580 \mathrm{ps}$.

Figure 3 Temperature contours of the case $E=5 \mathrm{~J} / \mathrm{m}^{2}, d=2 \mathrm{~nm}$. The temperature of the area under direct laser irradiation goes up very quickly. And the high temperature results in ablation. Consequently, a shock wave can be seen very clearly at $t=100 \mathrm{ps}$ and $t=$ 200 ps. Afterwards, the temperature in this area goes down in the recrystallization process. 
Figure 4 Atom number density contours of the case $E=5 \mathrm{~J} / \mathrm{m} 2, \mathrm{~d}=2 \mathrm{~nm}$. The whole domain is divided into $1 \times 10.2 \times 1(\mathrm{x} \times \mathrm{y} \times \mathrm{z}) \mathrm{nm} 3$ cells. The melting area is characterized by the uniform low atom number density. The distortion resulting from the stress wave accompanies the melting process. The recrystallization starts from $t=200$ ps. The distortion is remarkable in the place of defects. Argon lattice constant differs from the cell size in the contour calculation. Consequently, the atom number density in the cells changes periodically. The contours show patterned blocks of red and dark color and this periodicity is very helpful in demonstrating the defects (e.g. lattice bending and dislocation).

Figure 5 Crystallinity contours of the case $E=5 \mathrm{~J} / \mathrm{m}^{2}, d=2 \mathrm{~nm}$. The whole domain is divided into cells of size $1 \times 10.2 \times 1(x \times y \times z) \mathrm{nm}^{3}$. The blue region corresponds to liquid region. The melting area is characterized by low crystallinity (close to 0). And yellow and green correspond to the distorted solid area. The structure adjacent to the melting region is destructed as shown at $t=40 \mathrm{ps}$ and $100 \mathrm{ps}$. Both the liquid and solid can be present in the same cell, especially for the part close to the melting region. In the recrystallization process, the laser irradiation spot is the last part for full recovery in crystallinity. Distortion is characterized by the low crystallinity value spots as marked in the figure at $t=2580 \mathrm{ps}$.

Figure 6 Illustration of the tip confinement and tip-substrate distance effect on surface nanostructuring. For all cases, the laser fluence is $E=5 \mathrm{~J} / \mathrm{m} 2$. (a) Tip-substrate distance $\mathrm{d}=2 \mathrm{~nm}$. (b) Tip-substrate distance $\mathrm{d}=5 \mathrm{~nm}$. (c) Tip-substrate distance $\mathrm{d}=7$ nm. (d) Laser irradiation without a tip. Laser ablation and suspended clusters are observed. A strong spherical shock wave forms. For (a), (b) and (c), laser ablation is 
strongly blocked by the tip. The dynamics of the melting shows some different characteristics among the four cases.

Figure 7 (a)Final shape of the cone-shape crater in the substrate. The depth of the crater is not affected too much by the tip confinement. The side profile of the crater is slightly affected by the inclusion of tip confinement. The protrusion is different because of the existence of tip and the tip-substrate distance. (b) The number of atoms flying above $z=34.5 \mathrm{~nm}$ during the ablation process. Oscillation is observed due to the interaction of the tip and the substrate.

Figure 8 (a) $Z_{b}$ is the $z$ bottom position of the crater during the ablation process. $Z_{b}$ is going down quickly because of the laser ablation and also goes up quickly after the laser ablation. For the case of tip-free and $d=7 \mathrm{~nm}$, the crater bottom point is the deepest, while $d=1 \mathrm{~nm}$ case has the highest value at $t=500 \mathrm{ps}$. The crater bottom position for different cases is close to each other in the final stage. (b) $Z_{s l}$ is the $z$ position of the solid-liquid interface bottom point of the sample. $Z_{s l}$ goes down very quickly for all the cases in the first 40 ps. The solid-liquid interface bottom point then goes up slowly for all cases. The solid-liquid interface of the tip-free case goes up in the fastest speed. And it takes the longest time for $d=1 \mathrm{~nm}$ to finish the recrystallization process.

Figure 9 Comparison of final structure under different laser fluences. The time points for the 3 cases are: $E=2 \mathrm{~J} / \mathrm{m}^{2}, d=2 \mathrm{~nm}$, at $t=1440 \mathrm{ps} ; E=5 \mathrm{~J} / \mathrm{m}^{2}, d=2 \mathrm{~nm}$, at $t=2580 \mathrm{ps} ; E=$ $10 \mathrm{~J} / \mathrm{m}^{2}, d=2 \mathrm{~nm}$, at $t=4940 \mathrm{ps}$. (a) The final profile of the nanostructuring in final steady state. The crater size under laser fluence $E=10 \mathrm{~J} / \mathrm{m}^{2}$ is the biggest one and the laser fluence $E=2 \mathrm{~J} / \mathrm{m}^{2}$ gives the smallest crater. (b) Crystallinity of the final 
structure under different lase fluences. Distortion is clearly observed. The position of distortion for laser fluence $E=2 \mathrm{~J} / \mathrm{m}^{2}$ is higher than that of the laser fluence $E=5 \mathrm{~J} / \mathrm{m}^{2}$. The distortion is larger in size for laser fluence $E=10 \mathrm{~J} / \mathrm{m}^{2}$. This concludes when the laser fluence is becoming higher, larger and deeper distortion is formed within the substrate.

Figure 10 (a)Comparison of the profile and the final nanostructure surface to show the laser fluence effect. For high laser fluence $E=5 \mathrm{~J} / \mathrm{m}^{2}$, the width and height of the coneshape crater is bigger. And the protrusion is higher than that of $E=2 \mathrm{~J} / \mathrm{m}^{2}$. (b) Evolution of the solid-liquid interface bottom point $Z_{s l}$ under different laser fluences. For the case of $E=2 \mathrm{~J} / \mathrm{m}^{2}$, the melting depth is much smaller than the case of laser fluence $E=5 \mathrm{~J} / \mathrm{m}^{2}$. Also it takes a much shorter time for the crater to fully form and get stable for the case of $E=2 \mathrm{~J} / \mathrm{m}^{2}$.

Figure 11 (a) Tip profile at $t=1440 \mathrm{ps}$. For both cases, the tip-sample distance is $d=2 \mathrm{~nm}$. The comparison shows that for a laser fluence as high as $E=10 \mathrm{~J} / \mathrm{m}^{2}$, the tip apex is seriously distorted and the shape is more blunt than the case of $E=5 \mathrm{~J} / \mathrm{m}^{2}$. (b) Tip profile at $t=40$ ps. For both cases, the laser fluence is $5 \mathrm{~J} / \mathrm{m}^{2}$. The degree of tip distortion is more serious for $d=2 \mathrm{~nm}$ than $7 \mathrm{~nm}$.

Figure 12 (a)Variation of the position of the lowest point of the tip during surface nanostructuring. The tip moves up and down along the $z$ direction. (b) A window of size of $4 \times 10 \times 2 \mathrm{~nm}^{3}(x \times y \times z)$ beneath the tip is chosen as the area for temperature monitoring. For all the cases, the laser fluence is $E=5 \mathrm{~J} / \mathrm{m}^{2}$. The temperature in this window increases at first because of the laser ablation. Then it decreases since at the later time the ablated material deposits on the surface or they fly away from this 
window. 


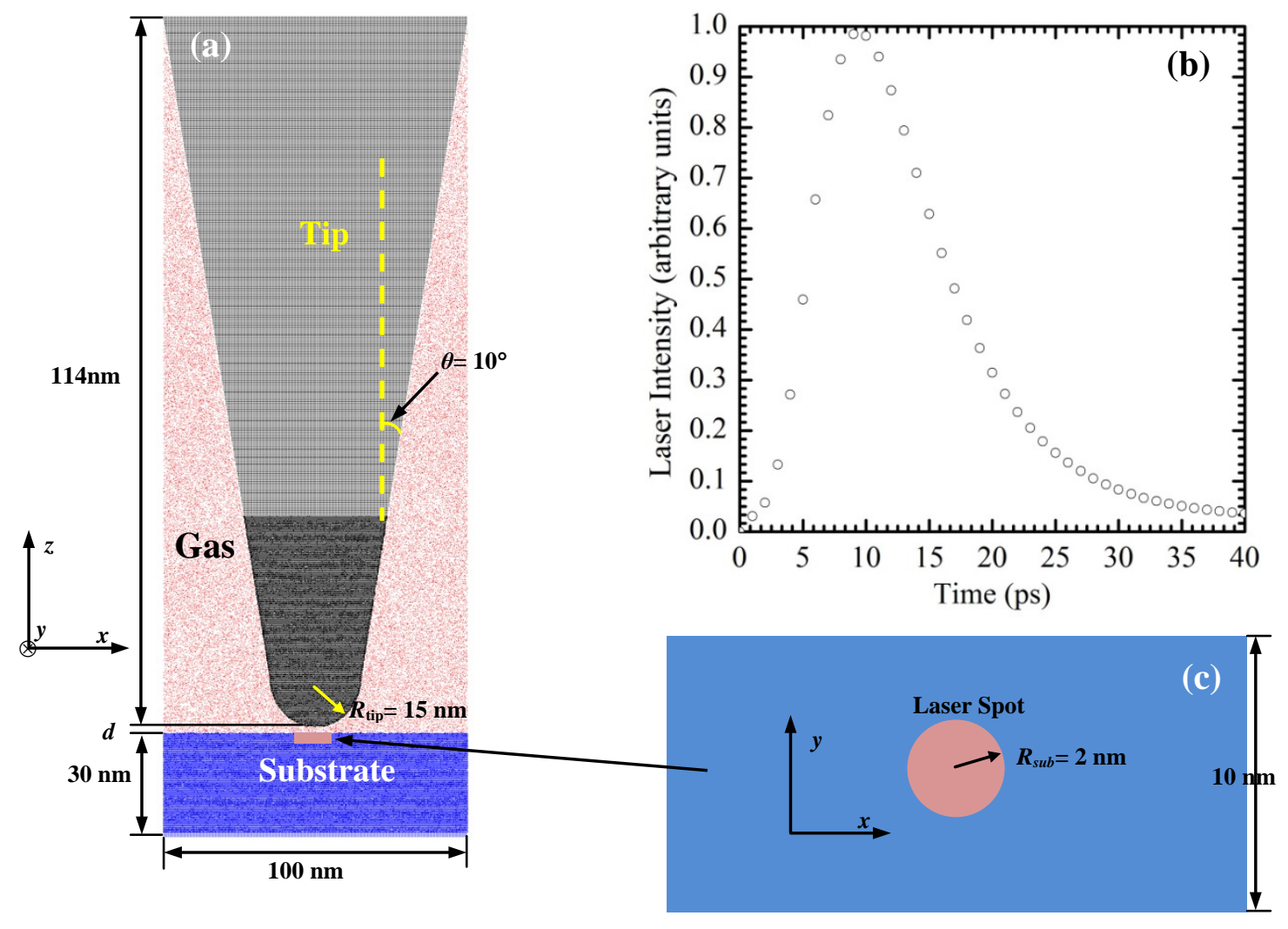

Fig.1(a) Physical model for simulating the tip-substrate nanostructuring process. The laser energy is focusedto a circular region with $R_{s u b}=2 \mathrm{~nm}$ in the substrate underneath the tip apex. The substrate is of size $100 \mathrm{~nm} \times 10 \mathrm{~nm} \times 30 \mathrm{~nm}(x \times y \times z)$. There are 3 important parameters for the tip construction: apex radius $R_{\text {tip }}=15 \mathrm{~nm}$, half taper angle $\theta=10^{\circ}$, length of tip $L=114 \mathrm{~nm}$. The atoms inside the tip at $z \geq 98.1 \mathrm{~nm}$ are removed and the actual shape in the regionz $\geq 98.1 \mathrm{~nm}$ is an empty cell surrounded by 5 walls ( $x, y$ direction and $z$ direction on the top) with a thickness of 1 nm. (b) Laser beam intensity distribution. The full width at half maximum (FWHM) of the incident laser beam intensity distribution is $11.5 \mathrm{ps}$ and peak occurs at $t=9 \mathrm{ps}$. (c) Profile of the laser spot. The spot is round with a radius of $R_{\text {sub }}=2 \mathrm{~nm}$. 

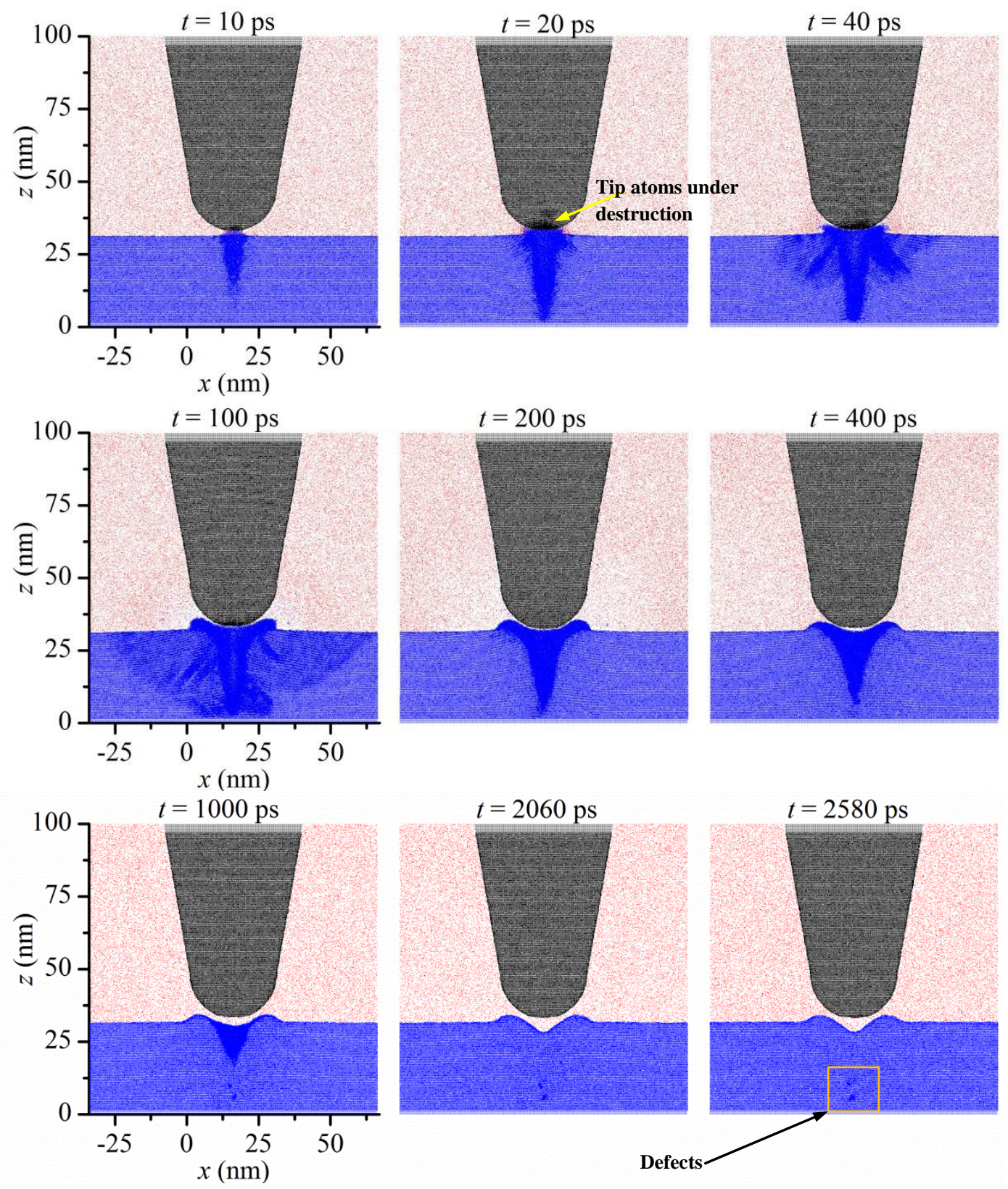

Fig. 2 Snapshots of the case $E=5 \mathrm{~J} / \mathrm{m}^{2}, d=2 \mathrm{~nm}$. Laser ablation starts from around $t=10 \mathrm{ps}$. Because of the confinement by the tip, the ablated material is prevented from moving freely in space. The apex of the tip is slightly distorted by the ablated-out substrate. Recrystallization ( $t=$ 200-2060 ps) is observed following the ablation. Finally, a steady cone-shape crater is formed. 
And at the same time, there are defects in the final sample structure at the bottom, as marked in the figure at $t=2580 \mathrm{ps}$. 

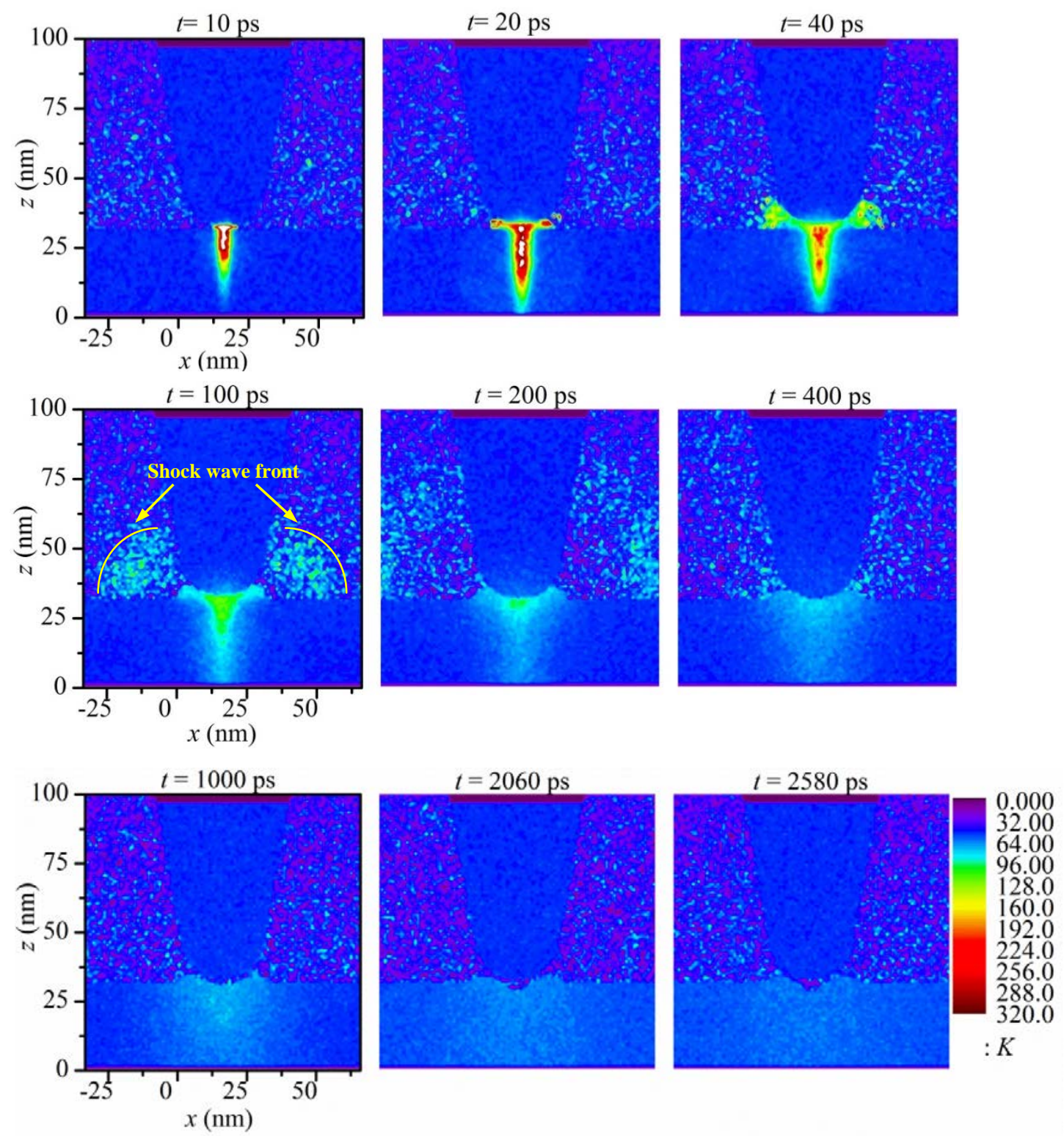

Fig. 3 Temperature contours of the case $E=5 \mathrm{~J} / \mathrm{m}^{2}, d=2 \mathrm{~nm}$. The temperature of the area under direct laser irradiation goes up very quickly. And the high temperature results in ablation. Consequently, a shock wave can be seen very clearly at $t=100 \mathrm{ps}$ and $t=200 \mathrm{ps}$. Afterwards, the temperature in this area goes down in the recrystallization process. 

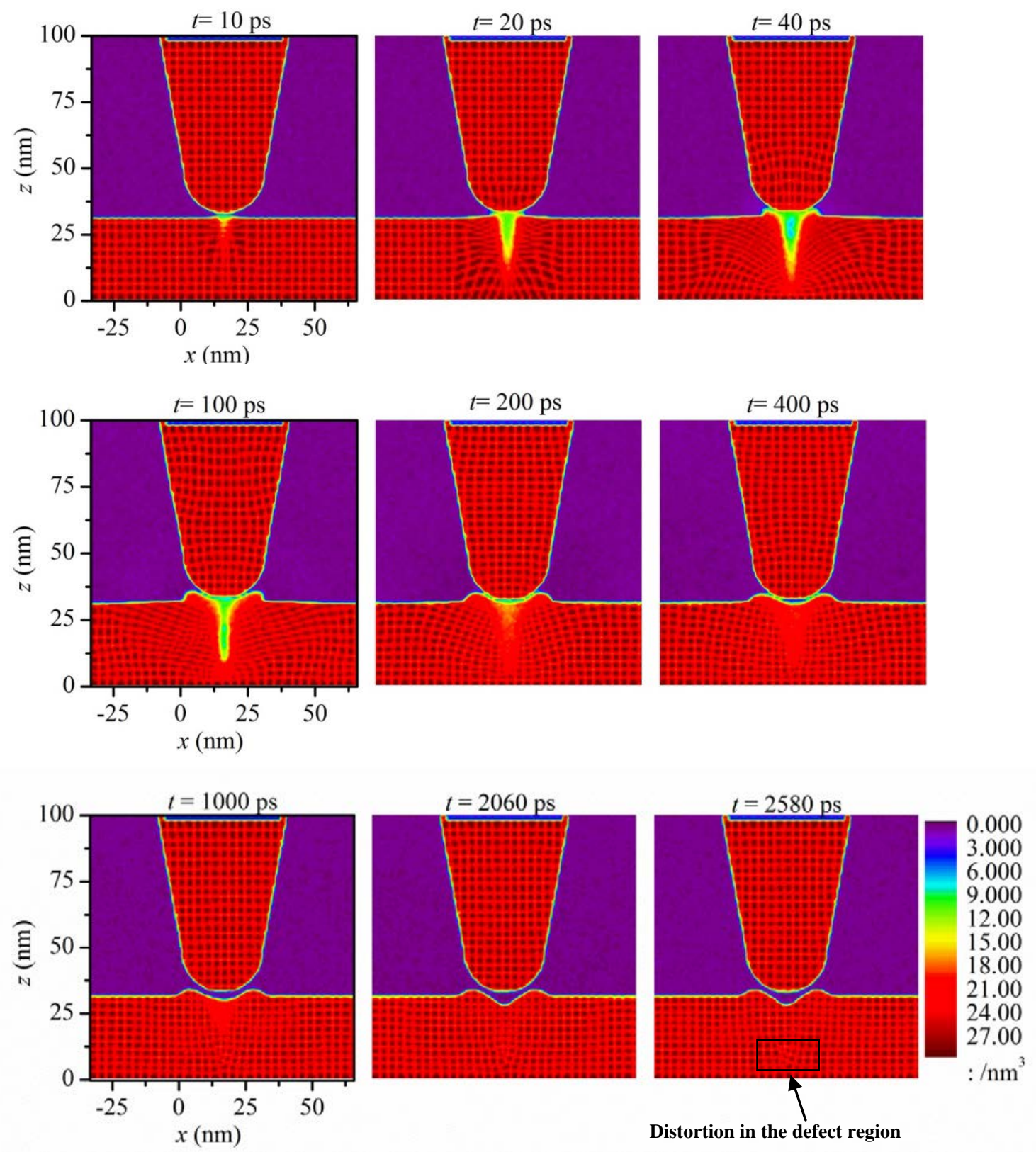

Fig. 4 Atomnumber density contours of the case $E=5 \mathrm{~J} / \mathrm{m}^{2}, d=2 \mathrm{~nm}$. The whole domain is divided into $1 \times 10.2 \times 1(x \times y \times z) \mathrm{nm}^{3}$ cells. The melting area is characterized by the uniform low atom number density. The distortion resulting from the stress wave accompanies the melting process. The recrystallization starts from $t=200 \mathrm{ps}$. The distortion is remarkable in the place of defects. Argon lattice constant differs from the cell size in the contour calculation. Consequently, 
the atom number density in the cells changes periodically. The contours show patterned blocks of red and dark color and this periodicity is very helpful in demonstrating the defects (e.g. lattice bending and dislocation). 

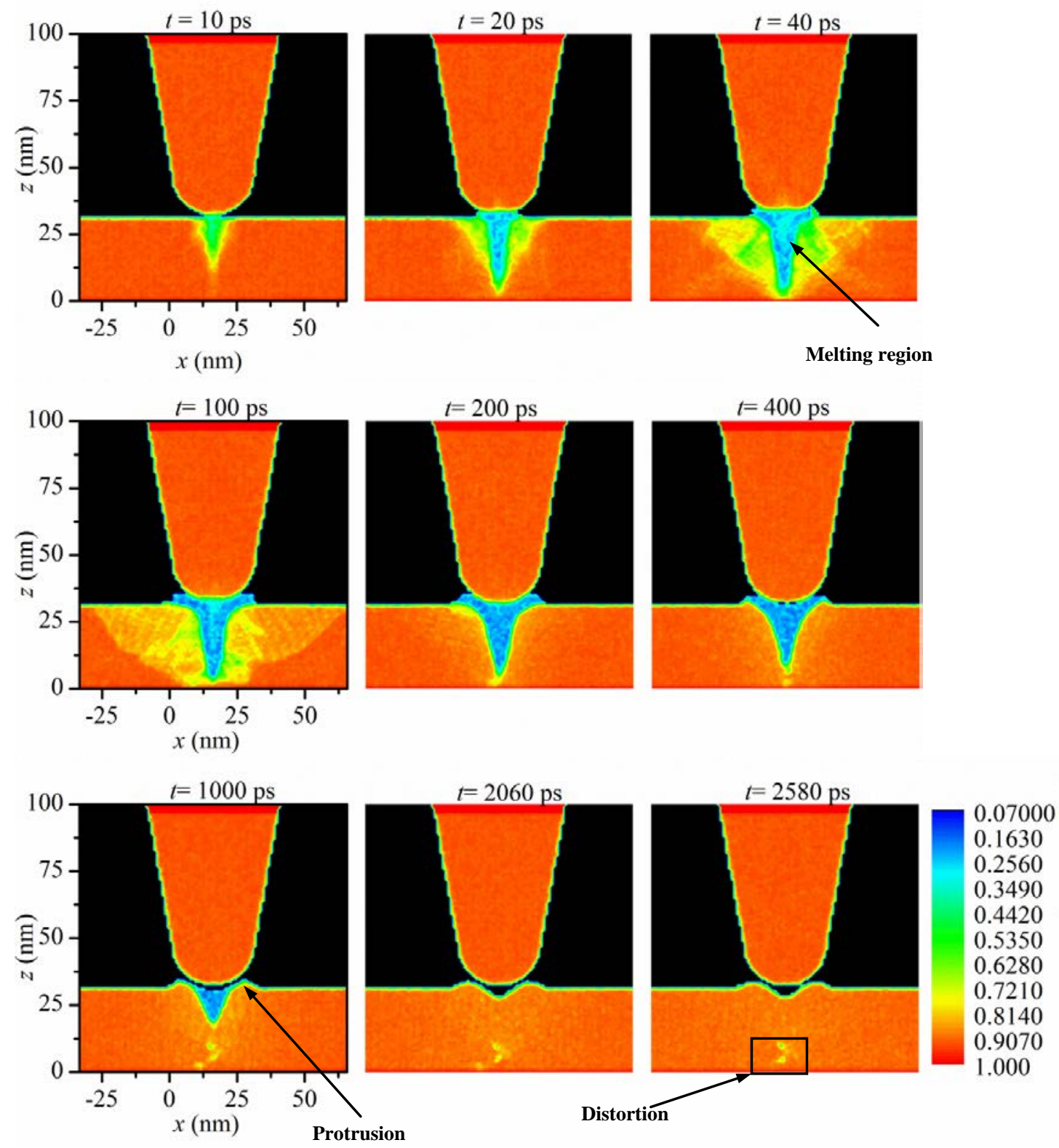

Fig. 5 Crystallinity contours of the case $E=5 \mathrm{~J} / \mathrm{m}^{2}, d=2 \mathrm{~nm}$. The whole domain is divided into cells of size $1 \times 10.2 \times 1(x \times y \times z) \mathrm{nm}^{3}$. The blue region corresponds to liquid region. The melting area is characterized by low crystallinity (close to 0 ). And yellow and green correspond to the distorted solid area. The structure adjacent to the melting region is destructed as shown at $t=40$ ps and 100 ps. Both the liquid and solid can be present in the same cell, especially for the part close to the melting region. In the recrystallization process, the laser irradiation spot is the last 
part for full recovery in crystallinity. Distortion is characterized by the low crystallinity value spots as marked in the figure at $t=2580 \mathrm{ps}$. 

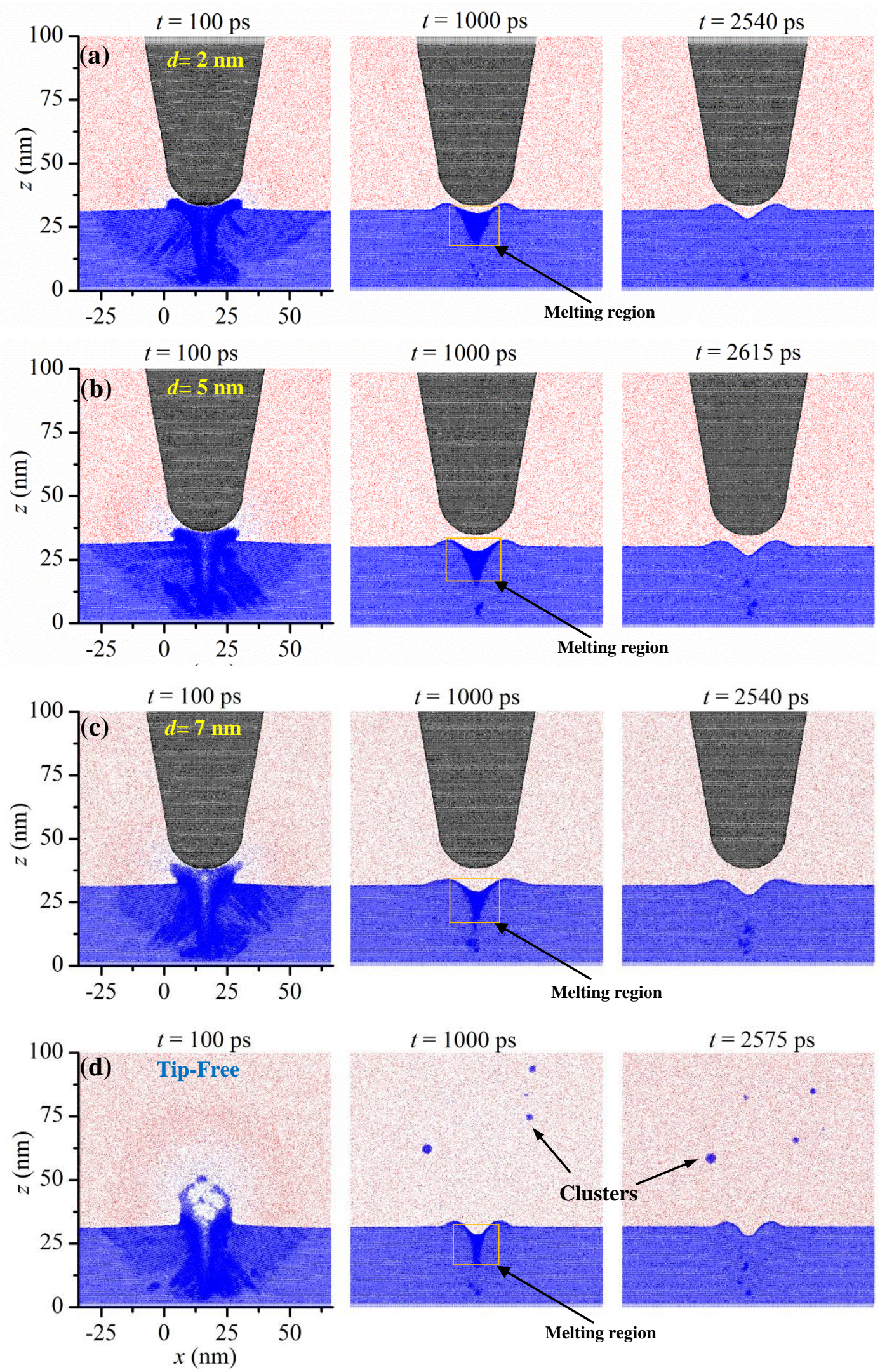
Fig. 6 Illustration of the tip confinement and tip-substrate distance effect on surface nanostructuring. For all cases, the laser fluence is $E=5 \mathrm{~J} / \mathrm{m}^{2}$. (a) Tip-substrate distance $d=2 \mathrm{~nm}$. (b) Tip-substrate distance $d=5 \mathrm{~nm}$. (c) Tip-substrate distance $d=7 \mathrm{~nm}$. (d) Laser irradiation without a tip. Laser ablation and suspended clusters are observed. A strong spherical shock wave forms. For (a), (b) and (c), laser ablation is strongly blocked by the tip. The dynamics of the melting shows some different characteristics among the four cases. 

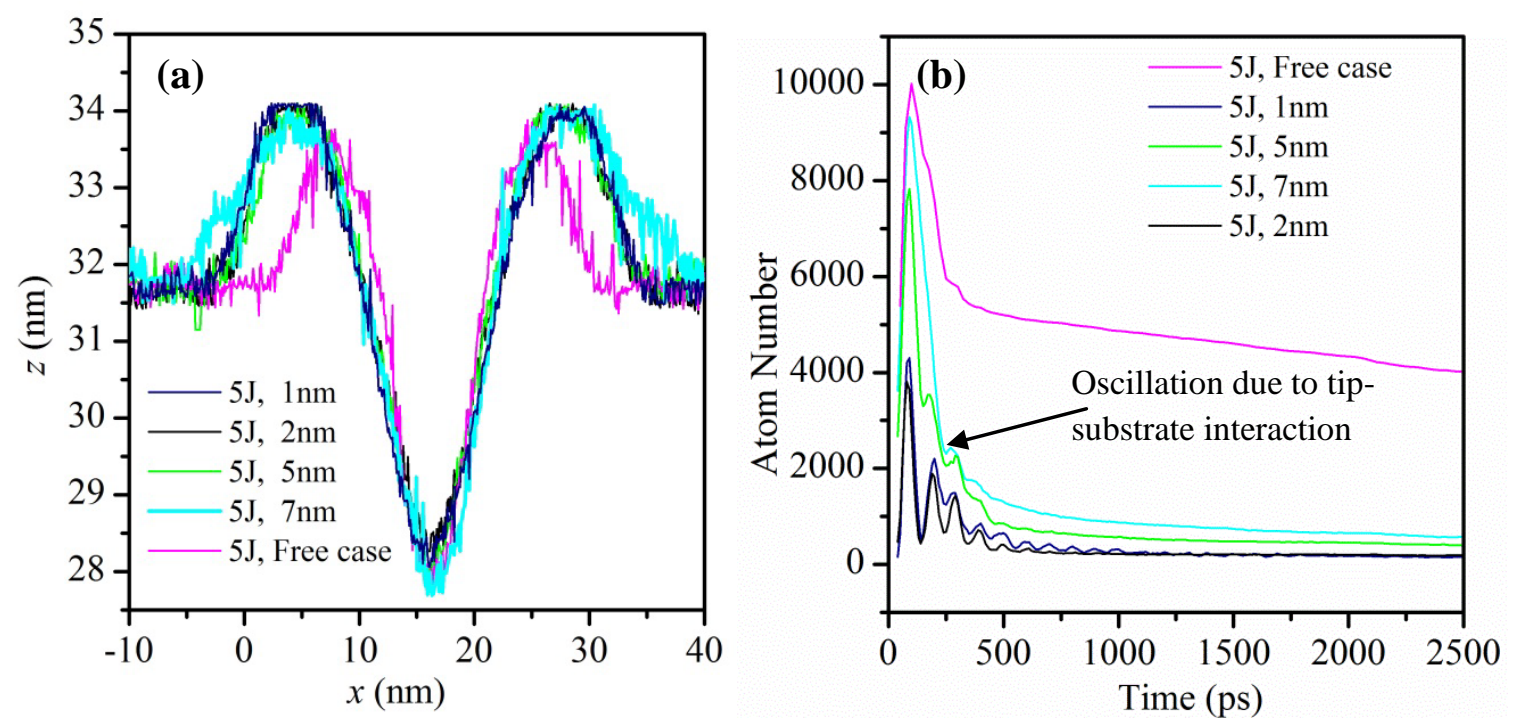

Fig. 7 (a)Final shape of the cone-shape crater in the substrate. The depth of the crater is not affected too much by the tip confinement. The side profile of the crater is slightly affected by the inclusion of tip confinement. The protrusion is different because of the existence of tip and the tip-substrate distance. (b) The number of atoms flying above $z=34.5 \mathrm{~nm}$ during the ablation process. Oscillation is observed due to the interaction of the tip and the substrate. 

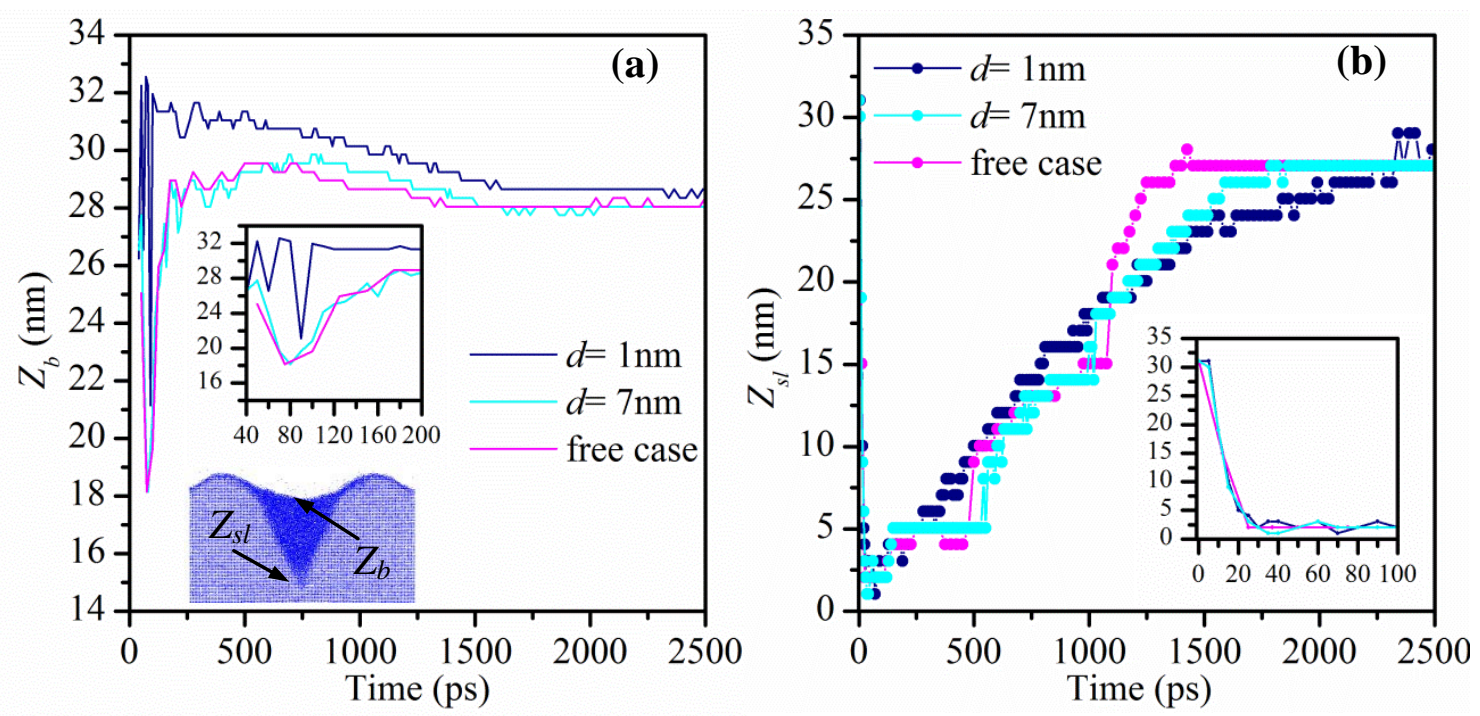

Fig. 8 (a) $Z_{b}$ is the $z$ bottom position of the crater during the ablation process. $Z_{b}$ is going down quickly because of the laser ablation and also goes up quickly after the laser ablation. For the case of tip-free and $d=7 \mathrm{~nm}$, the crater bottom point is the deepest, while $d=1 \mathrm{~nm}$ case has the highest value at $t=500 \mathrm{ps}$. The crater bottom position for different cases is close to each other in the final stage. (b) $Z_{s l}$ is the $z$ position of the solid-liquid interface bottom point of the sample. $Z_{s l}$ goes down very quickly for all the cases in the first 40 ps. The solid-liquid interface bottom point then goes up slowly for all cases. The solid-liquid interface of the tip-free case goes up in the fastest speed. And it takes the longest time for $d=1 \mathrm{~nm}$ to finish the recrystallization process. 

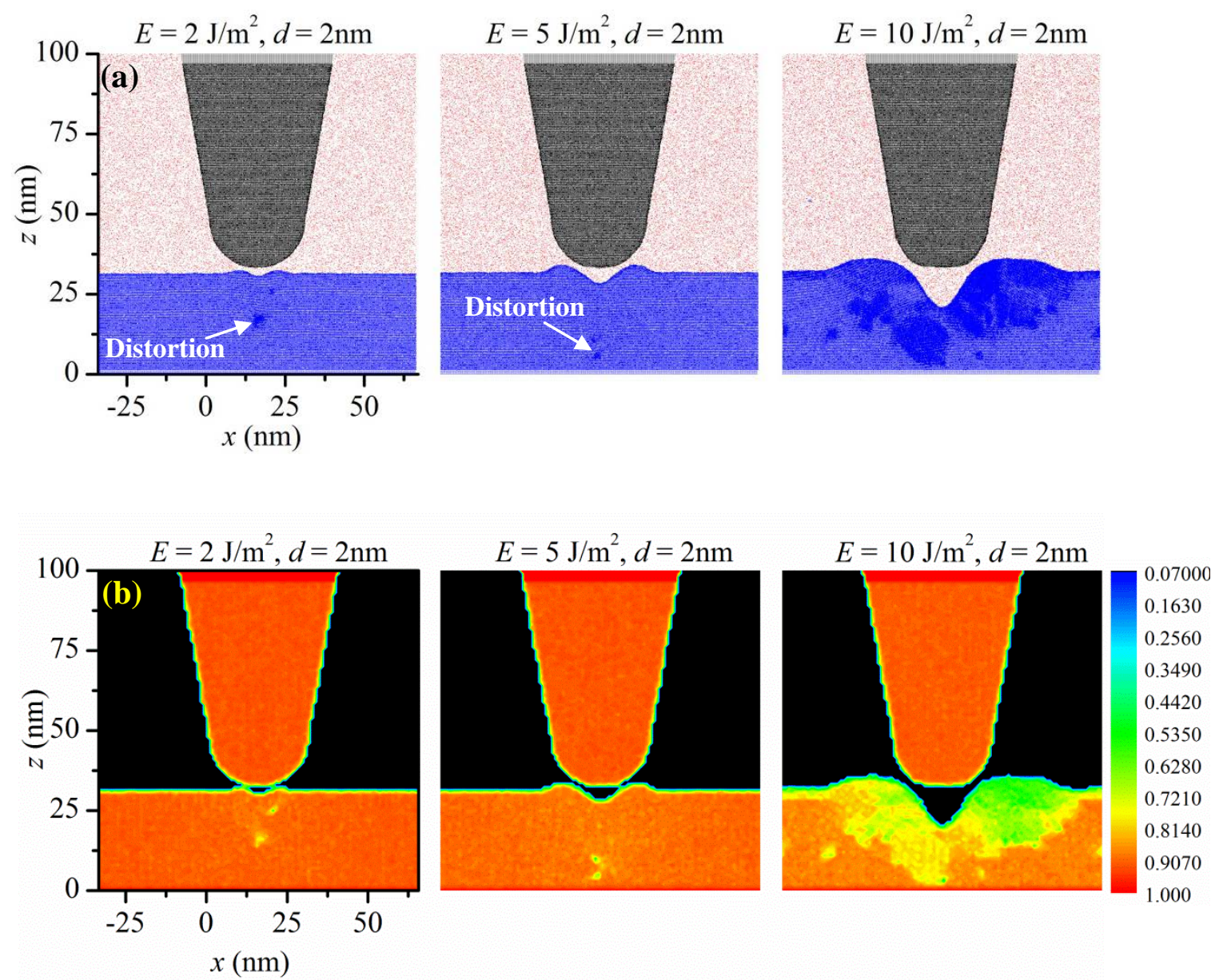

Fig. 9 Comparison of final structure under different laser fluences. The time points for the 3 cases are: $E=2 \mathrm{~J} / \mathrm{m}^{2}, d=2 \mathrm{~nm}$, at $t=1440 \mathrm{ps} ; E=5 \mathrm{~J} / \mathrm{m}^{2}, d=2 \mathrm{~nm}$, at $t=2580 \mathrm{ps} ; E=10 \mathrm{~J} / \mathrm{m}^{2}, d=2$ $\mathrm{nm}$, at $t=4940 \mathrm{ps}$. (a) The final profile of the nanostructuring in final steady state. The crater size under laser fluence $E=10 \mathrm{~J} / \mathrm{m}^{2}$ is the biggest one and the laser fluence $E=2 \mathrm{~J} / \mathrm{m}^{2}$ gives the smallest crater. (b) Crystallinity of the final structure under different lase fluences. Distortion is clearly observed. The position of distortion for laser fluence $E=2 \mathrm{~J} / \mathrm{m}^{2}$ is higher than that of the laser fluence $E=5 \mathrm{~J} / \mathrm{m}^{2}$. The distortion is larger in size for laser fluence $E=10 \mathrm{~J} / \mathrm{m}^{2}$. This concludes when the laser fluence is becoming higher, larger and deeper distortion is formed within the substrate. 

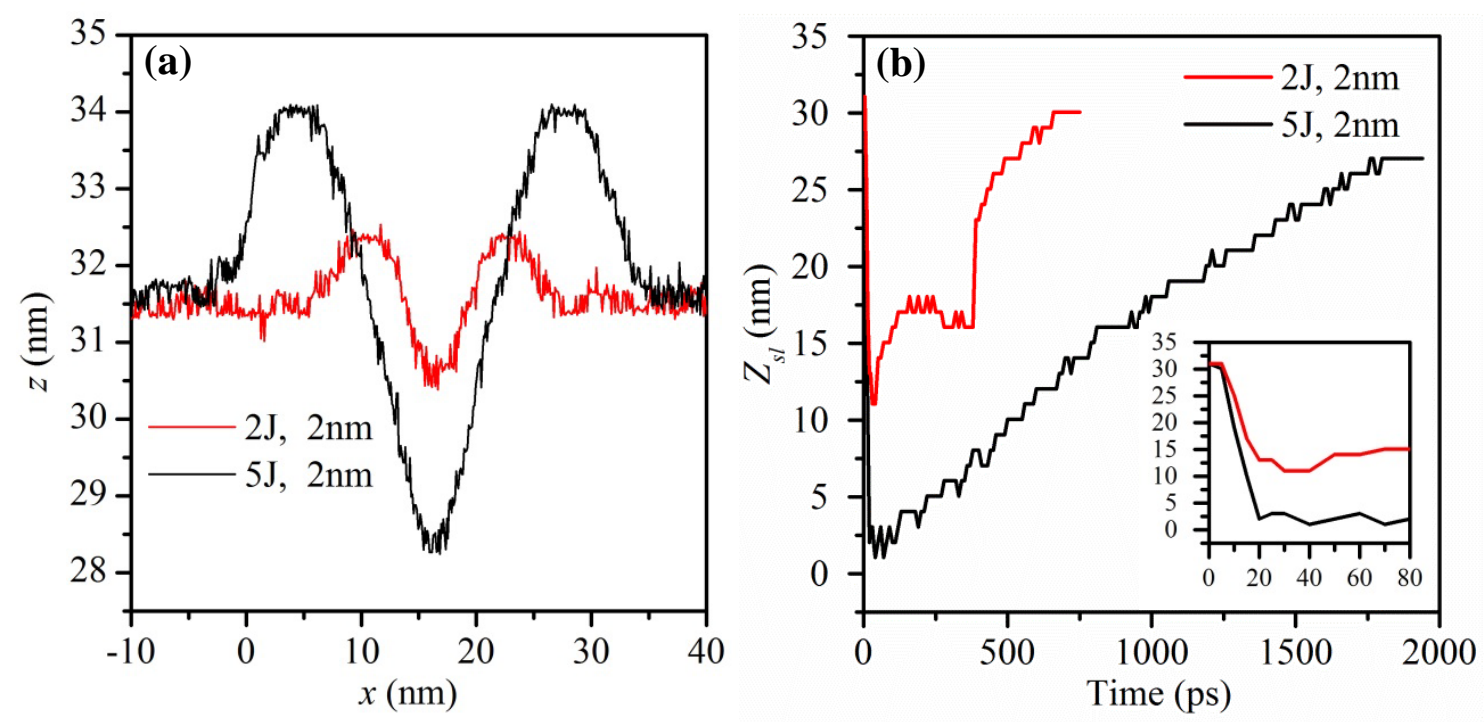

Fig. 10(a)Comparison of the profile and the final nanostructure surface to show the laser fluence effect. For high laser fluence $E=5 \mathrm{~J} / \mathrm{m}^{2}$, the width and height of the cone-shape crater is bigger. And the protrusion is higher than that of $E=2 \mathrm{~J} / \mathrm{m}^{2}$. (b) Evolution of the solid-liquid interface bottom point $Z_{s l}$ under different laser fluences. For the case of $E=2 \mathrm{~J} / \mathrm{m}^{2}$, the melting depth is much smaller than the case of laser fluence $E=5 \mathrm{~J} / \mathrm{m}^{2}$. Also it takes a much shorter time for the crater to fully form and get stable for the case of $E=2 \mathrm{~J} / \mathrm{m}^{2}$. 

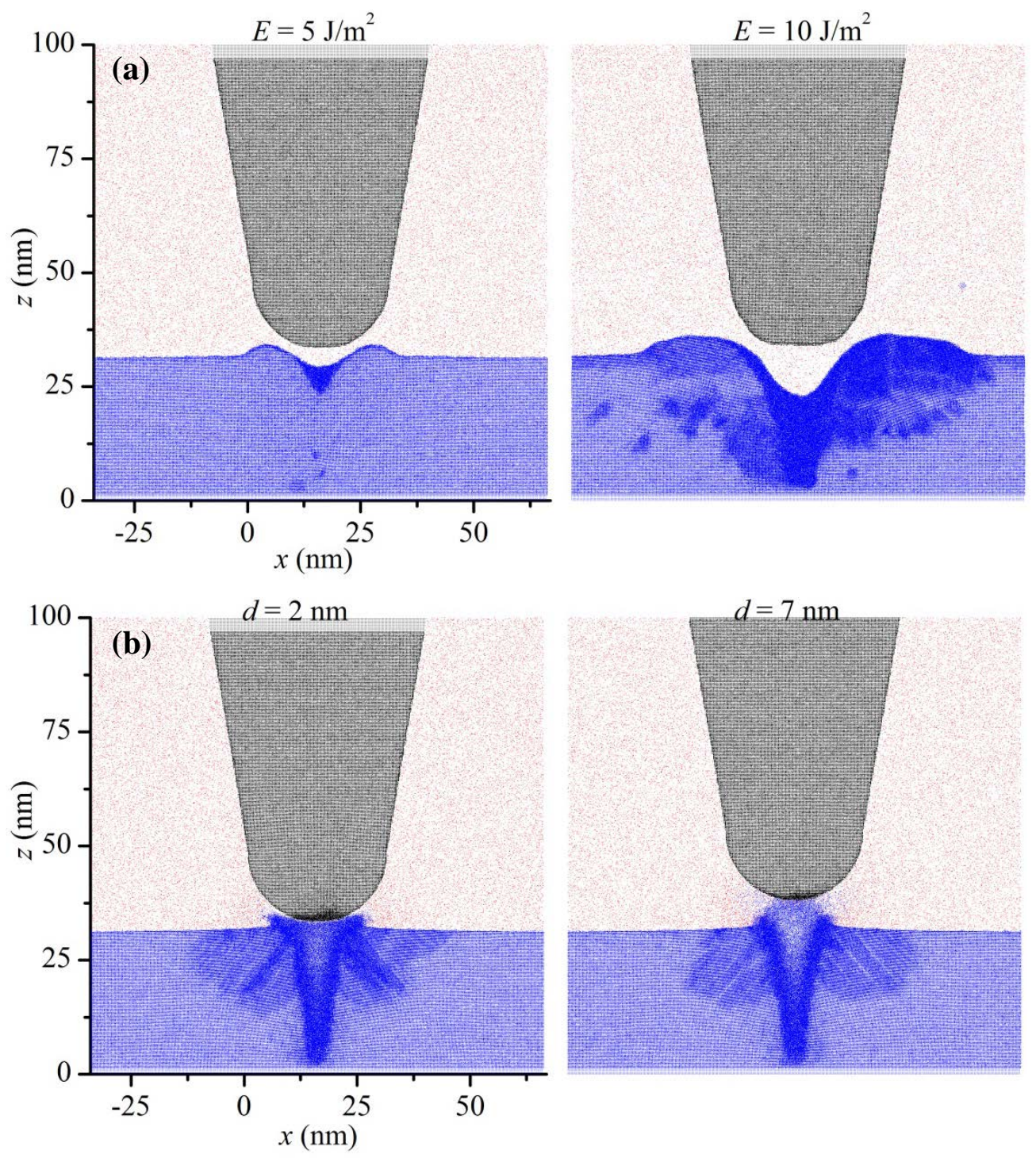

Fig. 11 (a) Tip profile at $t=1440$ ps. For both cases, the tip-sample distance is $d=2 \mathrm{~nm}$. The comparison shows that for a laser fluence as high as $E=10 \mathrm{~J} / \mathrm{m}^{2}$, the tip apex is seriously distorted and the shape is more blunt than the case of $E=5 \mathrm{~J} / \mathrm{m}^{2}$. (b) Tip profile at $t=40 \mathrm{ps}$. For both cases, the laser fluence is $5 \mathrm{~J} / \mathrm{m}^{2}$. The degree of tip distortion is more serious for $d=2 \mathrm{~nm}$ than $7 \mathrm{~nm}$. 

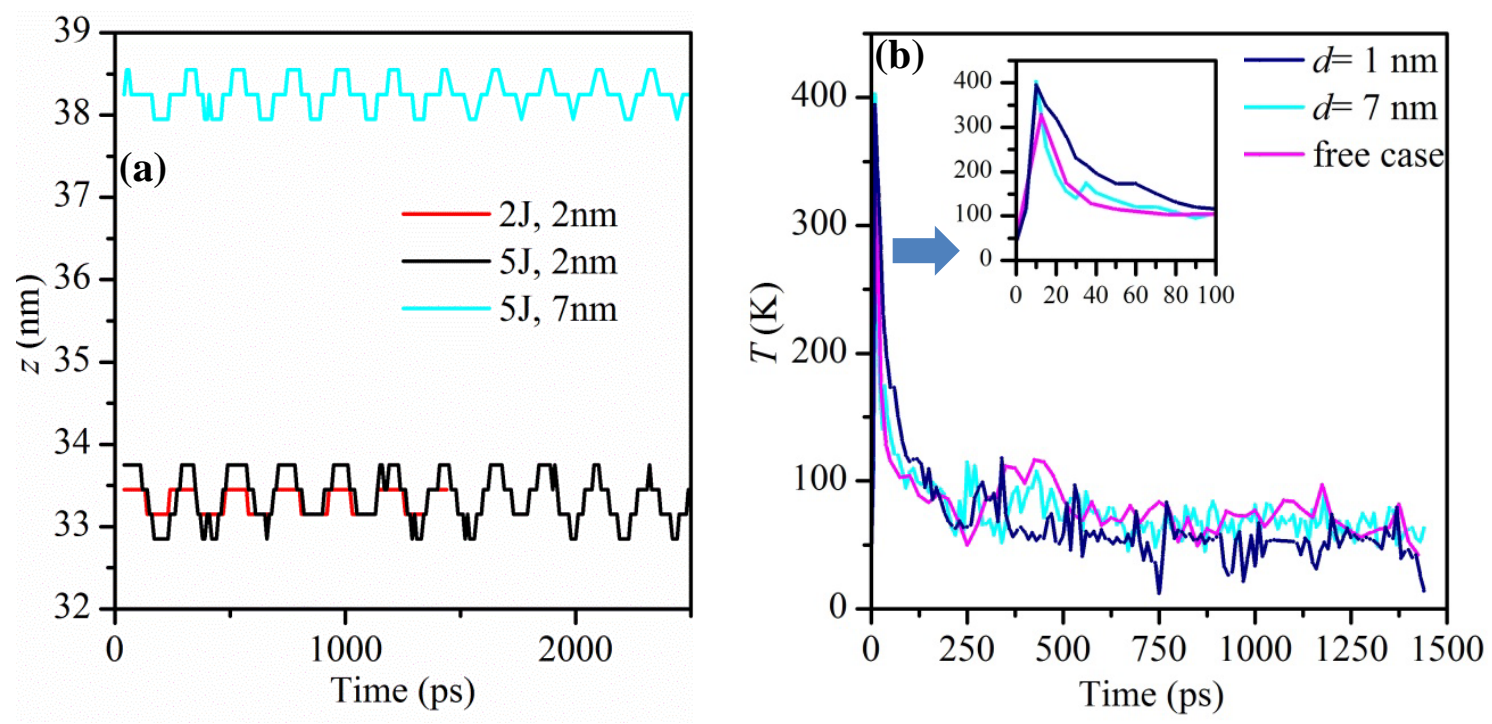

Fig. 12 (a)Variation ofthe position of the lowest point of the tip during surface nanostructuring. The tip moves up and down along the $z$ direction. (b) A window of size of $4 \times 10 \times 2 \mathrm{~nm}^{3}(x \times y \times z)$ beneath the tip is chosen as the area for temperature monitoring. For all the cases, the laser fluence is $E=5 \mathrm{~J} / \mathrm{m}^{2}$. The temperature in this window increases at first because of the laser ablation. Then it decreases since at the later time the ablated material deposits on the surface or they fly away from this window. 